九州大学学術情報リポジトリ

Kyushu University Institutional Repository

\title{
Construction of Integrals of Higher-Order Mappings
}

Maruno, Ken-ichi

Department of Mathematics, The University of Texas-Pan American | Faculty of Mathematics, Kyushu University

Quispel, G. Reinout W.

Department of Mathematics, La Trobe University | Centre of Excellence for Mathematics and Statistics of Complex Systems, La Trobe University

http://hdl. handle. net/2324/11858

出版情報: Journal of the Physical society of Japan. 75 (12), pp.123001-, 2006-11-27. The Physical Society of Japan

バージョン :

権利関係 : 


\title{
MHF Preprint Series
}

Kyushu University

21st Century COE Program

Development of Dynamic Mathematics with

High Functionality

\section{Construction of integrals of higher-order mappings}

\author{
K. Maruno \& G R W Quispel
}

MHF 2006-2

( Received January 6, 2006 )

Faculty of Mathematics

Kyushu University

Fukuoka, JAPAN 


\title{
LETTER TO THE EDITOR
}

\section{Construction of integrals of higher-order mappings}

\author{
Ken-ichi Maruno ${ }^{1}$ and G R W Quispel ${ }^{2,3}$ \\ ${ }^{1}$ Faculty of Mathematics, Kyushu University, Hakozaki, Higashi-ku, Fukuoka, 812-8581, \\ Japan \\ 2 Department of Mathematics, La Trobe University, Melbourne, Victoria 3086, Australia \\ ${ }^{3}$ Centre of Excellence for Mathematics and Statistics of Complex Systems, La Trobe \\ University, Melbourne, Victoria 3086, Australia
}

\begin{abstract}
We find that certain higher-order mappings arise as reductions of the integrable discrete AKP and BKP equations. Finding conservation laws for the AKP and BKP equations, we use these conservation laws to derive integrals of the associated reduced maps.
\end{abstract}

16 December 2005

PACS numbers: $02.30 . \mathrm{Ik}, 02.30 . \mathrm{Ks}$

To be submitted to :

\section{Introduction}

The search for discrete integrable systems has received a lot of attention in the past decade. This has resulted in the discovery of integrable mappings of the second-order, e.g. the QRT mapping [1], and discrete Painlevé equations [2]. Apart from second-order integrable mappings, the results for higher-order integrable mappings are few $[3,4,5,6,7,8,9,10,11]$. Discrete integrable systems have applications to various areas of physics, such as statistical mechanics, quantum gravity, and discrete analogues of integrable systems in classical mechanics and solid state physics. Here we study a novel class of higher-order integrable mappings which have bilinear forms.

As an example, we discuss the following 6th-order mapping:

$D x_{n+3} x_{n+2}^{2} x_{n+1}^{3} x_{n}^{3} x_{n-1}^{3} x_{n-2}^{2} x_{n-3}+A x_{n+2} x_{n+1}^{2} x_{n}^{2} x_{n-1}^{2} x_{n-2}+B x_{n+1} x_{n} x_{n-1}+C=0$.

(Here and below $A, B, C, D$ are arbitrary parameters). How can we obtain integrals for this mapping? In the paper [3], a method for construction of integrals was proposed and integrable third-order mappings which possess two integrals were obtained. However, this method is not applicable to higher-order mappings because this method uses some ansatz at first and needs the help of high performance computers. If we consider 6th-order, 8th-order and higher-order mappings, this method does not work, as current computer power is not sufficient [12].

In this Letter, we propose a systematic method to construct integrals for a class of higherorder integrable mappings without the help of computers. Our method proposed here is based 
on discrete bilinear forms related to the AKP and BKP soliton equations. Conservation laws for integrable partial difference equations have been studied in [13, 14].

\section{Conservation Laws for Discrete Bilinear Forms}

Before we discuss conservation laws for discrete systems, let us briefly recall conservation laws for continuous systems [15]. To this end, consider a (scalar) partial differential equation (PDE) $\Delta\left[x, u^{(i)}\right]=0$. A conservation law of such a PDE is a divergence expression

$$
\sum_{j} \frac{\partial P_{j}}{\partial x_{j}}=0
$$

which vanishes for all solutions of the given system. It follows that there exists a function $\Lambda$ (called the characteristic of the given conservation law) such that

$$
\sum_{j} \frac{\partial P_{j}}{\partial x_{j}}=\Lambda \Delta .
$$

Similarly, a conservation law of a scalar partial difference equation $\Delta\left[n, u_{n}\right]=0$ is an expression

$$
\sum_{j}\left(S_{j}-i d\right) P_{j}=0
$$

which vanishes for all solutions of the discrete system. (Here $S_{j}$ is a unit shift in the $n_{j}$ direction, and $\Delta\left[n, u_{n}\right]$ denotes a smooth function depending on $n, u_{n}$ and finitely many iterates of $u_{n}$ ). It follows again that there exists a function $\Lambda$ such that

$$
\sum_{j}\left(S_{j}-i d\right) P_{j}=\Lambda \Delta .
$$

We will call $\Lambda$ the characteristic of the discrete conservation law.

Here we give a list of characteristics of the discrete AKP and BKP equations.

\section{Discrete BKP equation}

The discrete BKP equation [16] is given by

$$
A \tau_{k+1, l, m} \tau_{k, l+1, m+1}+B \tau_{k, l+1, m} \tau_{k+1, l, m+1}+C \tau_{k, l, m+1} \tau_{k+1, l+1, m}+D \tau_{k, l, m} \tau_{k+1, l+1, m+1}=0 .
$$

We have found the following 12 explicit rational characteristics for the discrete BKP equation:

$$
\begin{aligned}
\Lambda_{1}= & A\left(\frac{\tau_{k-1, l+1, m+1}}{\tau_{k, l, m+1} \tau_{k, l+1, m+1} \tau_{k, l+1, m}}-\frac{\tau_{k+2, l, m}}{\tau_{k+1, l, m} \tau_{k+1, l+1, m} \tau_{k+1, l, m+1}}\right) \\
& +D\left(\frac{\tau_{k-1, l, m}}{\tau_{k, l, m} \tau_{k, l+1, m} \tau_{k, l, m+1}}-\frac{\tau_{k+2, l+1, m+1}}{\tau_{k+1, l+1, m+1} \tau_{k+1, l+1, m} \tau_{k+1, l, m+1}}\right), \\
\Lambda_{2}= & B\left(\frac{\tau_{k+1, l-1, m+1}}{\tau_{k+1, l, m} \tau_{k+1, l, m+1} \tau_{k, l, m+1}}-\frac{\tau_{k, l+2, m}}{\tau_{k, l+1, m} \tau_{k, l+1, m+1} \tau_{k+1, l+1, m}}\right) \\
& +D\left(\frac{\tau_{k, l-1, m}}{\tau_{k, l, m} \tau_{k, l, m+1} \tau_{k+1, l, m}}-\frac{\tau_{k+1, l+2, m+1}}{\tau_{k+1, l+1, m+1} \tau_{k, l+1, m+1} \tau_{k+1, l+1, m}}\right), \\
\Lambda_{3}= & C\left(\frac{\tau_{k+1, l+1, m-1}}{\tau_{k+1, l+1, m} \tau_{k, l+1, m} \tau_{k+1, l, m}}-\frac{\tau_{k, l, m+2}}{\tau_{k, l, m+1} \tau_{k, l+1, m+1} \tau_{k+1, l, m+1}}\right)
\end{aligned}
$$




$$
\begin{aligned}
& +D\left(\frac{\tau_{k, l, m-1}}{\tau_{k+1, l, m} \tau_{k, l+1, m} \tau_{k, l, m}}-\frac{\tau_{k+1, l+1, m+2}}{\tau_{k+1, l+1, m+1} \tau_{k, l+1, m+1} \tau_{k+1, l, m+1}}\right), \\
& \Lambda_{4}=C\left(\frac{\tau_{k, l-1, m+1}}{\tau_{k+1, l, m+1} \tau_{k, l, m+1} \tau_{k, l, m}}-\frac{\tau_{k+1, l+2, m}}{\tau_{k+1, l+1, m} \tau_{k, l+1, m} \tau_{k+1, l+1, m+1}}\right) \\
& +A\left(\frac{\tau_{k+1, l-1, m}}{\tau_{k+1, l, m} \tau_{k, l, m} \tau_{k+1, l, m+1}}-\frac{\tau_{k, l+2, m+1}}{\tau_{k, l+1, m+1} \tau_{k, l+1, m} \tau_{k+1, l+1, m+1}}\right), \\
& \Lambda_{5}=A\left(\frac{\tau_{k+1, l, m-1}}{\tau_{k+1, l+1, m} \tau_{k+1, l, m} \tau_{k, l, m}}-\frac{\tau_{k, l+1, m+2}}{\tau_{k, l+1, m+1} \tau_{k, l, m+1} \tau_{k+1, l+1, m+1}}\right) \\
& +B\left(\frac{\tau_{k, l+1, m-1}}{\tau_{k, l+1, m} \tau_{k, l, m} \tau_{k+1, l+1, m}}-\frac{\tau_{k+1, l, m+2}}{\tau_{k+1, l, m+1} \tau_{k, l, m+1} \tau_{k+1, l+1, m+1}}\right), \\
& \Lambda_{6}=B\left(\frac{\tau_{k-1, l+1, m}}{\tau_{k, l+1, m+1} \tau_{k, l+1, m} \tau_{k, l, m}}-\frac{\tau_{k+2, l, m+1}}{\tau_{k+1, l, m+1} \tau_{k+1, l, m} \tau_{k+1, l+1, m+1}}\right) \\
& +C\left(\frac{\tau_{k-1, l, m+1}}{\tau_{k, l, m+1} \tau_{k, l, m} \tau_{k, l+1, m+1}}-\frac{\tau_{k+2, l+1, m}}{\tau_{k+1, l+1, m} \tau_{k+1, l, m} \tau_{k+1, l+1, m+1}}\right), \\
& \Gamma_{1}=A(-1)^{k}\left(\frac{\tau_{k-1, l+1, m+1}}{\tau_{k, l, m+1} \tau_{k, l+1, m+1} \tau_{k, l+1, m}}+\frac{\tau_{k+2, l, m}}{\tau_{k+1, l, m} \tau_{k+1, l+1, m} \tau_{k+1, l, m+1}}\right) \\
& +D(-1)^{k}\left(\frac{\tau_{k-1, l, m}}{\tau_{k, l, m} \tau_{k, l+1, m} \tau_{k, l, m+1}}+\frac{\tau_{k+2, l+1, m+1}}{\tau_{k+1, l+1, m+1} \tau_{k+1, l+1, m} \tau_{k+1, l, m+1}}\right), \\
& \Gamma_{2}=B(-1)^{l}\left(\frac{\tau_{k+1, l-1, m+1}}{\tau_{k+1, l, m} \tau_{k+1, l, m+1} \tau_{k, l, m+1}}+\frac{\tau_{k, l+2, m}}{\tau_{k, l+1, m} \tau_{k, l+1, m+1} \tau_{k+1, l+1, m}}\right) \\
& +D(-1)^{l}\left(\frac{\tau_{k, l-1, m}}{\tau_{k, l, m} \tau_{k, l, m+1} \tau_{k+1, l, m}}+\frac{\tau_{k+1, l+2, m+1}}{\tau_{k+1, l+1, m+1} \tau_{k, l+1, m+1} \tau_{k+1, l+1, m}}\right), \\
& \Gamma_{3}=C(-1)^{m}\left(\frac{\tau_{k+1, l+1, m-1}}{\tau_{k+1, l+1, m} \tau_{k, l+1, m} \tau_{k+1, l, m}}+\frac{\tau_{k, l, m+2}}{\tau_{k, l, m+1} \tau_{k, l+1, m+1} \tau_{k+1, l, m+1}}\right) \\
& +D(-1)^{m}\left(\frac{\tau_{k, l, m-1}}{\tau_{k+1, l, m} \tau_{k, l+1, m} \tau_{k, l, m}}+\frac{\tau_{k+1, l+1, m+2}}{\tau_{k+1, l+1, m+1} \tau_{k, l+1, m+1} \tau_{k+1, l, m+1}}\right) \text {, } \\
& \Gamma_{4}=C(-1)^{l}\left(\frac{\tau_{k, l-1, m+1}}{\tau_{k+1, l, m+1} \tau_{k, l, m+1} \tau_{k, l, m}}+\frac{\tau_{k+1, l+2, m}}{\tau_{k+1, l+1, m} \tau_{k, l+1, m} \tau_{k+1, l+1, m+1}}\right) \\
& +A(-1)^{l}\left(\frac{\tau_{k+1, l-1, m}}{\tau_{k+1, l, m} \tau_{k, l, m} \tau_{k+1, l, m+1}}+\frac{\tau_{k, l+2, m+1}}{\tau_{k, l+1, m+1} \tau_{k, l+1, m} \tau_{k+1, l+1, m+1}}\right) \text {, } \\
& \Gamma_{5}=A(-1)^{m}\left(\frac{\tau_{k+1, l, m-1}}{\tau_{k+1, l+1, m} \tau_{k+1, l, m} \tau_{k, l, m}}+\frac{\tau_{k, l+1, m+2}}{\tau_{k, l+1, m+1} \tau_{k, l, m+1} \tau_{k+1, l+1, m+1}}\right) \\
& +B(-1)^{m}\left(\frac{\tau_{k, l+1, m-1}}{\tau_{k, l+1, m} \tau_{k, l, m} \tau_{k+1, l+1, m}}+\frac{\tau_{k+1, l, m+2}}{\tau_{k+1, l, m+1} \tau_{k, l, m+1} \tau_{k+1, l+1, m+1}}\right) \text {, } \\
& \Gamma_{6}=B(-1)^{k}\left(\frac{\tau_{k-1, l+1, m}}{\tau_{k, l+1, m+1} \tau_{k, l+1, m} \tau_{k, l, m}}+\frac{\tau_{k+2, l, m+1}}{\tau_{k+1, l, m+1} \tau_{k+1, l, m} \tau_{k+1, l+1, m+1}}\right) \\
& +C(-1)^{k}\left(\frac{\tau_{k-1, l, m+1}}{\tau_{k, l, m+1} \tau_{k, l, m} \tau_{k, l+1, m+1}}+\frac{\tau_{k+2, l+1, m}}{\tau_{k+1, l+1, m} \tau_{k+1, l, m} \tau_{k+1, l+1, m+1}}\right) .
\end{aligned}
$$


From these characteristics we can obtain the associated conservation laws, using eq.(2). For example, $P_{1}, P_{2}$ and $P_{3}$ associated to $\Lambda_{1}$ are

$$
\begin{aligned}
P_{1} & =-A^{2} \frac{\tau_{k-1, l+1, m+1} \tau_{k+1, l, m}}{\tau_{k+1, l+1, m} \tau_{k+1, l, m+1}}-D^{2} \frac{\tau_{k-1, l, m} \tau_{k+1, l+1, m+1}}{\tau_{k, l+1, m} \tau_{k, l, m+1}}-A B \frac{\tau_{k-1, l+1, m} \tau_{k+1, l, m}}{\tau_{k, l, m} \tau_{k, l+1, m}} \\
& -B D \frac{\tau_{k-1, l+1, m} \tau_{k+1, l+1, m+1}}{\tau_{k, l+1, m+1} \tau_{k, l+1, m}}-A C \frac{\tau_{k-1, l, m+1} \tau_{k+1, l, m}}{\tau_{k, l, m} \tau_{k, l, m+1}}-C D \frac{\tau_{k-1, l, m+1} \tau_{k+1, l+1, m+1}}{\tau_{k, l+1, m+1} \tau_{k, l, m+1}} \\
& -A D\left(\frac{\tau_{k-1, l, m} \tau_{k, l+1, m+1} \tau_{k+1, l, m}}{\tau_{k, l, m} \tau_{k, l+1, m} \tau_{k, l, m+1}}+\frac{\tau_{k, l, m} \tau_{k-1, l+1, m+1} \tau_{k+1, l+1, m+1}}{\tau_{k, l+1, m+1} \tau_{k, l+1, m} \tau_{k, l, m+1}}\right), \\
P_{2} & =A C \frac{\tau_{k+1, l, m} \tau_{k-1, l, m+1}}{\tau_{k, l, m+1} \tau_{k, l, m}}-B D \frac{\tau_{k-1, l, m} \tau_{k+1, l, m+1}}{\tau_{k, l, m+1} \tau_{k, l, m}} \\
P_{3} & =A B \frac{\tau_{k+1, l, m} \tau_{k-1, l+1, m}}{\tau_{k, l, m} \tau_{k, l+1, m}}-C D \frac{\tau_{k-1, l, m} \tau_{k+1, l+1, m}}{\tau_{k, l+1, m} \tau_{k, l, m}}
\end{aligned}
$$

\section{Discrete AKP equation}

The discrete AKP (Hirota-Miwa) equation $[17,16]$ is given by

$A \tau_{k+1, l, m} \tau_{k, l+1, m+1}+B \tau_{k, l+1, m} \tau_{k+1, l, m+1}+C \tau_{k, l, m+1} \tau_{k+1, l+1, m}=0$.

Note that the discrete AKP equation is the special case $D=0$ of the discrete BKP equation. The discrete AKP equation inherits the above 12 characteristics (with $D=0$ ) from the discrete BKP equation and we have found the following 2 additional characteristics:

$$
\begin{aligned}
& \Lambda_{7}=\frac{\tau_{k, l, m}}{\tau_{k+1, l, m} \tau_{k, l+1, m} \tau_{k, l, m+1}}-\frac{\tau_{k+1, l+1, m+1}}{\tau_{k, l+1, m+1} \tau_{k+1, l, m+1} \tau_{k+1, l+1, m}}, \\
& \Gamma_{7}=(-1)^{k+l+m}\left(\frac{\tau_{k, l, m}}{\tau_{k+1, l, m} \tau_{k, l+1, m} \tau_{k, l, m+1}}+\frac{\tau_{k+1, l+1, m+1}}{\tau_{k, l+1, m+1} \tau_{k+1, l, m+1} \tau_{k+1, l+1, m}}\right) .
\end{aligned}
$$

\section{Reduction to Finite Dimensional Mappings and Construction of their Integrals}

First example Consider the following 4th-order mapping:

$D x_{n+2} x_{n+1}^{2} x_{n}^{2} x_{n-1}^{2} x_{n-2}+A x_{n+1} x_{n} x_{n-1}+B+\frac{C}{x_{n}}=0$,

Using the transformation of the dependent variable

$$
x_{n}=\frac{\tau_{n+1} \tau_{n-1}}{\tau_{n}^{2}},
$$

we obtain a bilinear form

$$
D \tau_{n+3} \tau_{n-3}+A \tau_{n+2} \tau_{n-2}+B \tau_{n+1} \tau_{n-1}+C \tau_{n}^{2}=0 .
$$

This bilinear form is obtained from the discrete BKP equation by applying the reduction $\tau_{n} \equiv \tau_{Z_{1} k+Z_{2} l+Z_{3} m}$ where $Z_{1}=1, Z_{2}=2, Z_{3}=3$. Using the characteristics of the discrete BKP equation, we obtain the following integrating factors for the discrete bilinear form (6):

$$
\begin{aligned}
& \Lambda_{1}=A\left(\frac{\tau_{n+1}}{\tau_{n} \tau_{n+2} \tau_{n-1}}-\frac{\tau_{n-1}}{\tau_{n-2} \tau_{n} \tau_{n+1}}\right)+D\left(\frac{\tau_{n-4}}{\tau_{n-3} \tau_{n-1} \tau_{n}}-\frac{\tau_{n+4}}{\tau_{n+3} \tau_{n} \tau_{n+1}}\right)=-\Lambda_{6}, \\
& \Lambda_{2}=B\left(\frac{\tau_{n-1}}{\tau_{n-2} \tau_{n+1} \tau_{n}}-\frac{\tau_{n+1}}{\tau_{n-1} \tau_{n+2} \tau_{n}}\right)+D\left(\frac{\tau_{n-5}}{\tau_{n-3} \tau_{n} \tau_{n-2}}-\frac{\tau_{n+5}}{\tau_{n+3} \tau_{n+2} \tau_{n}}\right)=-\Lambda_{4},
\end{aligned}
$$




$$
\begin{aligned}
& \Lambda_{3}=C\left(\frac{\tau_{n-3}}{\tau_{n} \tau_{n-1} \tau_{n-2}}-\frac{\tau_{n+3}}{\tau_{n} \tau_{n+2} \tau_{n+1}}\right)+D\left(\frac{\tau_{n-6}}{\tau_{n-2} \tau_{n-1} \tau_{n-3}}-\frac{\tau_{n+6}}{\tau_{n+3} \tau_{n+2} \tau_{n+1}}\right)=\frac{A}{D} \Lambda_{4}+\frac{B}{D} \Lambda_{6}, \\
& \Lambda_{4}=C\left(\frac{\tau_{n-2}}{\tau_{n+1} \tau_{n} \tau_{n-3}}-\frac{\tau_{n+2}}{\tau_{n} \tau_{n-1} \tau_{n+3}}\right)+A\left(\frac{\tau_{n-4}}{\tau_{n-2} \tau_{n-3} \tau_{n+1}}-\frac{\tau_{n+4}}{\tau_{n+2} \tau_{n-1} \tau_{n+3}}\right), \\
& \Lambda_{5}=A\left(\frac{\tau_{n-5}}{\tau_{n} \tau_{n-2} \tau_{n-3}}-\frac{\tau_{n+5}}{\tau_{n+2} \tau_{n} \tau_{n+3}}\right)+B\left(\frac{\tau_{n-4}}{\tau_{n-1} \tau_{n-3} \tau_{n}}-\frac{\tau_{n+4}}{\tau_{n+1} \tau_{n} \tau_{n+3}}\right)=-\frac{A}{D} \Lambda_{4}-\frac{B}{D} \Lambda_{6}, \\
& \Lambda_{6}=B\left(\frac{\tau_{n-2}}{\tau_{n+2} \tau_{n-1} \tau_{n-3}}-\frac{\tau_{n+2}}{\tau_{n+1} \tau_{n-2} \tau_{n+3}}\right)+C\left(\frac{\tau_{n-1}}{\tau_{n} \tau_{n-3} \tau_{n+2}}-\frac{\tau_{n+1}}{\tau_{n} \tau_{n-2} \tau_{n+3}}\right) .
\end{aligned}
$$

It is confirmed by using the bilinear form (6) that the integrating factors $\Lambda_{1}, \Lambda_{2}, \Lambda_{3}$ and $\Lambda_{5}$ lead to the indicated linear combinations of $\Lambda_{4}$ and $\Lambda_{6}$. Note that the two integrating factors $\Lambda_{4}$ and $\Lambda_{6}$ are independent and that the characteristics $\Gamma_{n}$ of the discrete BKP equation do not reduce to integrating factors of (6). From the above integrating factors, we can make integrating factors in terms of the $x$-variable:

$$
\begin{aligned}
\tilde{\Lambda}_{4} & =\tau_{n-1} \tau_{n+1} \Lambda_{4}=C\left(\frac{1}{x_{n-1} x_{n-2}}-\frac{1}{x_{n+2} x_{n+1}}\right)+A\left(x_{n-2} x_{n-3}-x_{n+3} x_{n+2}\right), \\
\tilde{\Lambda}_{6} & =\tau_{n-1} \tau_{n+1} \Lambda_{6} \\
& =B\left(\frac{1}{x_{n+1} x_{n} x_{n-1} x_{n-2}}-\frac{1}{x_{n+2} x_{n+1} x_{n} x_{n-1}}\right)+C\left(\frac{1}{x_{n+1} x_{n} x_{n-1}^{2} x_{n-2}}-\frac{1}{x_{n+2} x_{n+1}^{2} x_{n} x_{n-1}}\right) .
\end{aligned}
$$

We then obtain the following two integrals:

$$
\begin{aligned}
Q_{4} & =C D x_{n+2} x_{n+1}^{2} x_{n}^{2} x_{n-1}-A D x_{n+3} x_{n+2}^{2} x_{n+1}^{2} x_{n}^{2} x_{n-1}^{2} x_{n-2} \\
& -A^{2}\left(x_{n+3} x_{n+2} x_{n+1} x_{n} x_{n-1}+x_{n+2} x_{n+1} x_{n} x_{n-1} x_{n-2}\right)-C^{2}\left(\frac{1}{x_{n+2} x_{n+1} x_{n}}+\frac{1}{x_{n+1} x_{n} x_{n-1}}\right) \\
& -B C\left(\frac{1}{x_{n+2} x_{n+1}}+\frac{1}{x_{n+1} x_{n}}+\frac{1}{x_{n} x_{n-1}}\right)-A B \sum_{j=0}^{4} x_{n+3-j} x_{n+2-j}-A C \sum_{j=0}^{3} \frac{x_{n+3-j} x_{n+2-j}}{x_{n-j}}, \\
Q_{6} & =B D x_{n+2} x_{n+1} x_{n} x_{n-1}+C D\left(x_{n+2} x_{n+1} x_{n}+x_{n+1} x_{n} x_{n-1}\right)-A B\left(\frac{1}{x_{n+2}}+\frac{1}{x_{n+1}}+\frac{1}{x_{n}}+\frac{1}{x_{n-1}}\right) \\
& -A C\left(\frac{1}{x_{n+2} x_{n+1}}+\frac{1}{x_{n+1} x_{n}}+\frac{1}{x_{n} x_{n-1}}\right)-\frac{C^{2}}{x_{n+2} x_{n+1} x_{n} x_{n-1}}-\frac{1}{x_{n+2} x_{n+1}^{2} x_{n}^{2} x_{n-1}} \\
& -B C\left(\frac{1}{x_{n+2} x_{n+1} x_{n}^{2} x_{n-1}}+\frac{1}{x_{n+2} x_{n+1}^{2} x_{n} x_{n-1}}\right) .
\end{aligned}
$$

It is not difficult to show that $Q_{4}$ and $Q_{6}$ are functionally independent.

In the special case $D=0$, the fourth-order mapping (5) reduces to the second-order mapping

$$
A x_{n+1} x_{n} x_{n-1}+B+\frac{C}{x_{n}}=0,
$$

which is a special case of the QRT mapping [1]. Using the transformation of the dependent variable

$$
x_{n}=\frac{\tau_{n+1} \tau_{n-1}}{\tau_{n}^{2}}
$$


we obtain a bilinear form

$$
A \tau_{n+2} \tau_{n-2}+B \tau_{n+1} \tau_{n-1}+C \tau_{n}^{2}=0 .
$$

This bilinear form is obtained from the discrete AKP equation by applying the reduction $\tau_{n} \equiv \tau_{Z_{1} k+Z_{2} l+Z_{3} m}$ where $Z_{1}=1, Z_{2}=2, Z_{3}=3$. Using the characteristics of the discrete AKP equation, we obtain the following integrating factors for the discrete bilinear form (8):

$$
\begin{aligned}
& \Lambda_{1}=\left(\frac{\tau_{n+1}}{\tau_{n} \tau_{n+2} \tau_{n-1}}-\frac{\tau_{n-1}}{\tau_{n-2} \tau_{n} \tau_{n+1}}\right), \\
& \Lambda_{2}=\left(\frac{\tau_{n-1}}{\tau_{n-2} \tau_{n+1} \tau_{n}}-\frac{\tau_{n+1}}{\tau_{n-1} \tau_{n+2} \tau_{n}}\right)=-\Lambda_{1}, \\
& \Lambda_{3}=\left(\frac{\tau_{n-3}}{\tau_{n} \tau_{n-1} \tau_{n-2}}-\frac{\tau_{n+3}}{\tau_{n} \tau_{n+2} \tau_{n+1}}\right)=\frac{C}{A} \Lambda_{1}, \\
& \Lambda_{4}=C\left(\frac{\tau_{n-2}}{\tau_{n+1} \tau_{n} \tau_{n-3}}-\frac{\tau_{n+2}}{\tau_{n} \tau_{n-1} \tau_{n+3}}\right)+A\left(\frac{\tau_{n-4}}{\tau_{n-2} \tau_{n-3} \tau_{n+1}}-\frac{\tau_{n+4}}{\tau_{n+2} \tau_{n-1} \tau_{n+3}}\right)=B \Lambda_{1}, \\
& \Lambda_{5}=A\left(\frac{\tau_{n-5}}{\tau_{n} \tau_{n-2} \tau_{n-3}}-\frac{\tau_{n+5}}{\tau_{n+2} \tau_{n} \tau_{n+3}}\right)+B\left(\frac{\tau_{n-4}}{\tau_{n-1} \tau_{n-3} \tau_{n}}-\frac{\tau_{n+4}}{\tau_{n+1} \tau_{n} \tau_{n+3}}\right)=-\frac{C^{2}}{A} \Lambda_{1}, \\
& \Lambda_{6}=B\left(\frac{\tau_{n-2}}{\tau_{n+2} \tau_{n-1} \tau_{n-3}}-\frac{\tau_{n+2}}{\tau_{n+1} \tau_{n-2} \tau_{n+3}}\right)+C\left(\frac{\tau_{n-1}}{\tau_{n} \tau_{n-3} \tau_{n+2}}-\frac{\tau_{n+1}}{\tau_{n} \tau_{n-2} \tau_{n+3}}\right)=-A \Lambda_{1}, \\
& \Lambda_{7}=\frac{\tau_{n-3}}{\tau_{n-2} \tau_{n-1} \tau_{n}}-\frac{C}{\tau_{n+2} \tau_{n+1} \tau_{n}}=\frac{C}{A} \Lambda_{1} .
\end{aligned}
$$

There is only one independent integrating factor, $\Lambda_{1}$. From $\Lambda_{1}$, we can make an integrating factors in terms of the $x$-variable:

$$
\tilde{\Lambda}_{1}=\tau_{n+1} \tau_{n-1} \Lambda_{1}=\frac{1}{x_{n+1}}-\frac{1}{x_{n-1}} .
$$

We then obtain the following integral:

$$
Q_{1}=-A x_{n+1} x_{n}+B\left(\frac{1}{x_{n+1}}+\frac{1}{x_{n}}\right)+\frac{C}{x_{n+1} x_{n}} .
$$

\section{Second example}

As a second example, let us discuss the following 6th-order mapping

$D x_{n+3} x_{n+2}^{2} x_{n+1}^{3} x_{n}^{3} x_{n-1}^{3} x_{n-2}^{2} x_{n-3}+A x_{n+2} x_{n+1}^{2} x_{n}^{2} x_{n-1}^{2} x_{n-2}+B x_{n+1} x_{n} x_{n-1}+C=0$.

Using the transformation of the dependent variable

$$
x_{n}=\frac{\tau_{n+1} \tau_{n-1}}{\tau_{n}^{2}}
$$

we obtain a bilinear form

$$
A \tau_{n+3} \tau_{n-3}+B \tau_{n+2} \tau_{n-2}+C \tau_{n+1} \tau_{n-1}+D \tau_{n+4} \tau_{n-4}=0
$$

This bilinear form is obtained from the discrete BKP equation by applying the reduction $\tau_{n} \equiv \tau_{Z_{1} k+Z_{2} l+Z_{3} m}$ where $Z_{1}=1, Z_{2}=2, Z_{3}=5$ or $Z_{1}=1, Z_{2}=3, Z_{3}=4$. Using the 
characteristics of the discrete BKP equation, we obtain the following integrating factors for the discrete bilinear form (10):

$$
\begin{aligned}
& \Lambda_{1}=A\left(\frac{\tau_{n+2}}{\tau_{n+1} \tau_{n+3} \tau_{n-2}}-\frac{\tau_{n-2}}{\tau_{n-3} \tau_{n-1} \tau_{n+2}}\right)+D\left(\frac{\tau_{n-5}}{\tau_{n-4} \tau_{n-2} \tau_{n+1}}-\frac{\tau_{n+5}}{\tau_{n-1} \tau_{n+2} \tau_{n+4}}\right)=-\Lambda_{6}, \\
& \Lambda_{2}=B\left(\frac{\tau_{n}}{\tau_{n-3} \tau_{n+2} \tau_{n+1}}-\frac{\tau_{n}}{\tau_{n-2} \tau_{n+3} \tau_{n-1}}\right)+D\left(\frac{\tau_{n-6}}{\tau_{n-4} \tau_{n-3} \tau_{n+1}}-\frac{\tau_{n+6}}{\tau_{n-1} \tau_{n+3} \tau_{n+4}}\right)=\Lambda_{4} \text {, } \\
& \Lambda_{3}=C\left(\frac{\tau_{n-6}}{\tau_{n-1} \tau_{n-2} \tau_{n-3}}-\frac{\tau_{n+6}}{\tau_{n+1} \tau_{n+3} \tau_{n+2}}\right)+D\left(\frac{\tau_{n-9}}{\tau_{n-4} \tau_{n-3} \tau_{n-2}}-\frac{\tau_{n+9}}{\tau_{n+2} \tau_{n+3} \tau_{n+4}}\right)=-\Lambda_{5} \text {, } \\
& \Lambda_{4}=C\left(\frac{\tau_{n-1}}{\tau_{n+2} \tau_{n+1} \tau_{n-4}}-\frac{\tau_{n+1}}{\tau_{n-1} \tau_{n-2} \tau_{n+4}}\right)+A\left(\frac{\tau_{n-5}}{\tau_{n-3} \tau_{n-4} \tau_{n+2}}-\frac{\tau_{n+5}}{\tau_{n+3} \tau_{n-2} \tau_{n+4}}\right) \text {, } \\
& \Lambda_{5}=A\left(\frac{\tau_{n-8}}{\tau_{n-1} \tau_{n-3} \tau_{n-4}}-\frac{\tau_{n+8}}{\tau_{n+3} \tau_{n+1} \tau_{n+4}}\right)+B\left(\frac{\tau_{n-7}}{\tau_{n-2} \tau_{n-4} \tau_{n-1}}-\frac{\tau_{n+7}}{\tau_{n+2} \tau_{n+1} \tau_{n+4}}\right) \text {, } \\
& \Lambda_{6}=B\left(\frac{\tau_{n-3}}{\tau_{n+3} \tau_{n-2} \tau_{n-4}}-\frac{\tau_{n+3}}{\tau_{n+2} \tau_{n-3} \tau_{n+4}}\right)+C\left(\frac{\tau_{n}}{\tau_{n+1} \tau_{n-4} \tau_{n+3}}-\frac{\tau_{n}}{\tau_{n-1} \tau_{n-3} \tau_{n+4}}\right) .
\end{aligned}
$$

It is confirmed by using the bilinear form (10) that integrating factors $\Lambda_{1}, \Lambda_{2}$ and $\Lambda_{3}$ lead to $\Lambda_{6}, \Lambda_{4}$ and $\Lambda_{5}$ respectively. Note that the 3 integrating factors $\Lambda_{4}, \Lambda_{5}$ and $\Lambda_{6}$ are independent and that the characteristics $\Gamma_{n}$ of the discrete BKP equation do not reduce to integrating factors of (10). From the above integrating factors, we can make integrating factors in terms of the $x$-variable:

$$
\begin{aligned}
& \tilde{\Lambda}_{4}= \tau_{n+1} \tau_{n-1} \Lambda_{4} \\
&= \frac{C}{x_{n+1} x_{n}^{2} x_{n-1}}\left(\frac{1}{x_{n-1}^{2} x_{n-2}^{2} x_{n-3}}-\frac{1}{x_{n+3} x_{n+2}^{2} x_{n+1}^{2}}\right)+\frac{A}{x_{n+1} x_{n} x_{n-1}}\left(x_{n-3} x_{n-4}-x_{n+4} x_{n+3}\right), \\
& \tilde{\Lambda}_{5}= \tau_{n+1} \tau_{n-1} \Lambda_{5}=A x_{n}\left(x_{n-1}^{2} x_{n-2}^{3} x_{n-3}^{4} x_{n-4}^{4} x_{n-5}^{3} x_{n-6}^{2} x_{n-7}-x_{n+7} x_{n+6}^{2} x_{n+5}^{3} x_{n+4}^{4} x_{n+3}^{4} x_{n+2}^{3} x_{n+1}^{2}\right) \\
& \quad B x_{n}\left(x_{n-1}^{2} x_{n-2}^{3} x_{n-3}^{3} x_{n-4}^{3} x_{n-5}^{2} x_{n-6}-x_{n+6} x_{n+5}^{2} x_{n+4}^{3} x_{n+3}^{3} x_{n+2}^{3} x_{n+1}^{2}\right), \\
& \tilde{\Lambda}_{6}= \tau_{n+1} \tau_{n-1} \Lambda_{6}=\frac{B}{x_{n+2} x_{n+1}^{2} x_{n}^{2} x_{n-1}^{2} x_{n-2}}\left(\frac{1}{x_{n-3}}-\frac{1}{x_{n+3}}\right) \\
& \quad+\frac{C}{x_{n+2} x_{n+1}^{2} x_{n}^{3} x_{n-1}^{2} x_{n-2}}\left(\frac{1}{x_{n-1} x_{n-2} x_{n-3}}-\frac{1}{x_{n+3} x_{n+2} x_{n+1}}\right) .
\end{aligned}
$$

We then obtain the following three integrals $\ddagger$ :

$$
\begin{aligned}
Q_{4} & =C D\left(x_{n+3} x_{n+2}^{2} x_{n+1}^{2} x_{n}+x_{n+2} x_{n+1}^{2} x_{n}^{2} x_{n-1}+x_{n+1} x_{n}^{2} x_{n-1}^{2} x_{n-2}\right) \\
& -A D x_{n+4} x_{n+3}^{2} x_{n+2}^{2} x_{n+1}^{2} x_{n}^{2} x_{n-1}^{2} x_{n-2}^{2} x_{n-3}-A^{2} x_{n+3} x_{n+2} x_{n+1} x_{n} x_{n-1} x_{n-2}\left(x_{n+4}+x_{n-3}\right) \\
& -B C \sum_{j=0}^{2} \frac{1}{x_{n+3-j} x_{n+2-j}^{2} x_{n+1-j}^{2} x_{n-j}}-A B \sum_{j=0}^{6} x_{n+4-j} x_{n+3-j} \\
& -C^{2}\left(\frac{1}{x_{n+3} x_{n+2}^{2} x_{n+1}^{3} x_{n}^{2} x_{n-1}}+\frac{1}{x_{n+2} x_{n+1}^{2} x_{n}^{3} x_{n-1}^{2} x_{n-2}}\right)-A C \sum_{j=0}^{3} \frac{x_{n+4-j} x_{n+3-j}}{x_{n+1-j} x_{n-j} x_{n-1-j}}, \\
Q_{5} & =A D \sum_{j=0}^{3} x_{n+7-j} x_{n+6-j}^{2} x_{n+5-j}^{3} x_{n+4-j}^{4} x_{n+3-j}^{5} x_{n+2-j}^{5} x_{n+1-j}^{5} x_{n-j}^{4} x_{n-1-j}^{3} x_{n-2-j}^{2} x_{n-3-j}
\end{aligned}
$$

$\$$ Note that the map (9) can be used to eliminate e.g. $x_{n+4}$ and $x_{n-3}$ from $Q_{4}$. Similarly for $Q_{5}$ and $Q_{6}$. 


$$
\begin{aligned}
& +B D \sum_{j=0}^{2} x_{n+6-j} x_{n+5-j}^{2} x_{n+4-j}^{3} x_{n+3-j}^{4} x_{n+2-j}^{4} x_{n+1-j}^{5} x_{n-j}^{4} x_{n-1-j}^{3} x_{n-2-j}^{2} x_{n-3-j} \\
& +A^{2} \sum_{j=0}^{4} x_{n+7-j} x_{n+6-j}^{2} x_{n+5-j}^{3} x_{n+4-j}^{4} x_{n+3-j}^{4} x_{n+2-j}^{4} x_{n+1-j}^{4} x_{n-j}^{3} x_{n-1-j}^{2} x_{n-2-j} \\
& +A B \sum_{j=0}^{4} x_{n+6-j} x_{n+5-j}^{2} x_{n+4-j}^{3} x_{n+3-j}^{3} x_{n+2-j}^{4} x_{n+1-j}^{4} x_{n-j}^{3} x_{n-1-j}^{2} x_{n-2-j} \\
& +A B \sum_{j=0}^{4} x_{n+7-j} x_{n+6-j}^{2} x_{n+5-j}^{3} x_{n+4-j}^{4} x_{n+3-j}^{4} x_{n+2-j}^{3} x_{n+1-j}^{3} x_{n-j}^{2} x_{n-1-j} \\
& +B^{2} \sum_{j=0}^{5} x_{n+6-j} x_{n+5-j}^{2} x_{n+4-j}^{3} x_{n+3-j}^{3} x_{n+2-j}^{3} x_{n+1-j}^{3} x_{n-j}^{2} x_{n-1-j} \\
& +B C \sum_{j=0}^{5} x_{n+6-j} x_{n+5-j}^{2} x_{n+4-j}^{3} x_{n+3-j}^{3} x_{n+2-j}^{3} x_{n+1-j}^{2} x_{n-j} \\
& +A C \sum_{j=0}^{6} x_{n+7-j} x_{n+6-j}^{2} x_{n+5-j}^{3} x_{n+4-j}^{4} x_{n+3-j}^{4} x_{n+2-j}^{3} x_{n+1-j}^{2} x_{n-j}, \\
& Q_{6}=B D x_{n+3} x_{n+2} x_{n+1} x_{n} x_{n-1} x_{n-2}+C D \sum_{j=0}^{3} x_{n+3-j} x_{n+2-j} x_{n+1-j} \\
& -A B \sum_{j=0}^{5} \frac{1}{x_{n+3-j}}-A C \sum_{j=0}^{2} \frac{1}{x_{n+3-j} x_{n+2-j} x_{n+1-j} x_{n-j}} \\
& -\frac{B^{2}}{x_{n+3} x_{n+2} x_{n+1} x_{n} x_{n-1} x_{n-2}}-\frac{C^{2}}{x_{n+3} x_{n+2}^{2} x_{n+1}^{3} x_{n}^{3} x_{n-1}^{2} x_{n-2}} \\
& -\frac{B C}{x_{n+3} x_{n+2} x_{n+1}^{2} x_{n}^{2} x_{n-1}^{2} x_{n-2}}-\frac{B C}{x_{n+3} x_{n+2}^{2} x_{n+1}^{2} x_{n}^{2} x_{n-1} x_{n-2}} \text {. }
\end{aligned}
$$

Using e.g. Mathematica, one can show that $Q_{4}, Q_{5}$ and $Q_{6}$ are functionally independent.

We consider the 4th-order mapping

$$
A x_{n+2} x_{n+1}^{2} x_{n}^{2} x_{n-1}^{2} x_{n-2}+B x_{n+1} x_{n} x_{n-1}+C=0 .
$$

This mapping is the special case $D=0$ of the 6th-order mapping (9). Applying

$$
x_{n}=\frac{\tau_{n+1} \tau_{n-1}}{\tau_{n}^{2}},
$$

we have a bilinear form

$$
A \tau_{n+3} \tau_{n-3}+B \tau_{n+2} \tau_{n-2}+C \tau_{n+1} \tau_{n-1}=0 .
$$

This bilinear form is obtained from the discrete AKP equation by applying the reduction $\tau_{n} \equiv \tau_{Z_{1} k+Z_{2} l+Z_{3} m}$ where $Z_{1}=1, Z_{2}=2, Z_{3}=5$ or $Z_{1}=1, Z_{2}=3, Z_{3}=4$. Using the characteristics of the discrete AKP equation, we obtain the following integrating factors for the discrete bilinear form (12):

$$
\Lambda_{1}=\frac{\tau_{n+2}}{\tau_{n+1} \tau_{n+3} \tau_{n-2}}-\frac{\tau_{n-2}}{\tau_{n-3} \tau_{n-1} \tau_{n+2}},
$$




$$
\begin{aligned}
\Lambda_{2}= & \frac{\tau_{n}}{\tau_{n-3} \tau_{n+2} \tau_{n+1}}-\frac{\tau_{n}}{\tau_{n-2} \tau_{n+3} \tau_{n-1}}, \\
\Lambda_{3}= & \frac{\tau_{n-6}}{\tau_{n-1} \tau_{n-2} \tau_{n-3}}-\frac{\tau_{n+6}}{\tau_{n+1} \tau_{n+3} \tau_{n+2}}=-\frac{C^{2}}{A^{2}} \Lambda_{1}-\frac{B^{2} C}{A^{3}} \Lambda_{2}, \\
\Lambda_{4}= & C\left(\frac{\tau_{n-1}}{\tau_{n+2} \tau_{n+1} \tau_{n-4}}-\frac{\tau_{n+1}}{\tau_{n-1} \tau_{n-2} \tau_{n+4}}\right)+A\left(\frac{\tau_{n-5}}{\tau_{n-3} \tau_{n-4} \tau_{n+2}}-\frac{\tau_{n+5}}{\tau_{n+3} \tau_{n-2} \tau_{n+4}}\right)=-B \Lambda_{2}, \\
\Lambda_{5}= & A\left(\frac{\tau_{n-8}}{\tau_{n-1} \tau_{n-3} \tau_{n-4}}-\frac{\tau_{n+8}}{\tau_{n+3} \tau_{n+1} \tau_{n+4}}\right)+B\left(\frac{\tau_{n-7}}{\tau_{n-2} \tau_{n-4} \tau_{n-1}}-\frac{\tau_{n+7}}{\tau_{n+2} \tau_{n+1} \tau_{n+4}}\right) \\
= & \frac{C^{3}}{A^{2}} \Lambda_{1}+\frac{B^{2} C^{2}}{A^{3}} \Lambda_{2}, \\
\Lambda_{6}= & B\left(\frac{\tau_{n-3}}{\tau_{n+3} \tau_{n-2} \tau_{n-4}}-\frac{\tau_{n+3}}{\tau_{n+2} \tau_{n-3} \tau_{n+4}}\right)+C\left(\frac{\tau_{n}}{\tau_{n+1} \tau_{n-4} \tau_{n+3}}-\frac{\tau_{n}}{\tau_{n-1} \tau_{n-3} \tau_{n+4}}\right)=-A \Lambda_{1}, \\
\Lambda_{7}= & \frac{\tau_{n-4}}{\tau_{n-3} \tau_{n-2} \tau_{n+1}}-\frac{C}{\tau_{n+3} \tau_{n+2} \tau_{n-1}}=-\frac{1}{A} \Lambda_{2} .
\end{aligned}
$$

It is confirmed by using the bilinear form (12) that the two integrating factors $\Lambda_{1}$ and $\Lambda_{2}$ are independent and the characteristics $\Gamma_{n}$ of the discrete AKP equation do not reduce to integrating factors of (12). From the above integrating factors, we can make integrating factors in terms of the $x$-variable:

$$
\begin{aligned}
& \tilde{\Lambda}_{1}=\tau_{n+1} \tau_{n-1} \Lambda_{1}=\frac{1}{x_{n+1} x_{n} x_{n-1}}\left(\frac{1}{x_{n+2}}-\frac{1}{x_{n-2}}\right) \\
& \tilde{\Lambda}_{2}=\tau_{n+1} \tau_{n-1} \Lambda_{2}=\frac{1}{x_{n+1} x_{n}^{2} x_{n-1}}\left(\frac{1}{x_{n-1} x_{n-2}}-\frac{1}{x_{n+2} x_{n+1}}\right) .
\end{aligned}
$$

We then obtain the following two integrals:

$$
\begin{aligned}
& Q_{1}=-A x_{n+2} x_{n+1} x_{n} x_{n-1}+B\left(\frac{1}{x_{n+2}}+\frac{1}{x_{n+1}}+\frac{1}{x_{n}}+\frac{1}{x_{n-1}}\right)+\frac{C}{x_{n+2} x_{n+1} x_{n} x_{n-1}}, \\
& Q_{2}=A\left(x_{n+2} x_{n+1}+x_{n+1} x_{n}+x_{n} x_{n-1}\right)-B\left(\frac{1}{x_{n+2} x_{n+1} x_{n}}+\frac{1}{x_{n+1} x_{n} x_{n-1}}\right)-\frac{C}{x_{n+2} x_{n+1}^{2} x_{n}^{2} x_{n-1}} .
\end{aligned}
$$

We note that the map (11) preserves the symplectic structure

$$
\left(\begin{array}{cccc}
0 & x_{n-2} x_{n-1} & -x_{n-2} x_{n} & x_{n-2} x_{n+1} \\
-x_{n-1} x_{n-2} & 0 & x_{n-1} x_{n} & -x_{n-1} x_{n+1} \\
x_{n} x_{n-2} & -x_{n} x_{n-1} & 0 & x_{n} x_{n+1} \\
-x_{n+1} x_{n-2} & x_{n+1} x_{n-1} & -x_{n+1} x_{n} & 0
\end{array}\right),
$$

and that the two integrals $Q_{1}$ and $Q_{2}$ are in involution w.r.t. this structure, giving an independent confirmation of the integrability of the map (11).

\section{Conclusions}

We have studied a class of integrable mappings which have bilinear forms. We have proposed a method to construct integrals of these higher-order integrable maps. The key to the construction are the conservation laws of the discrete bilinear forms of the associated AKP and BKP equations. Note that, generalizing the examples in this Letter, we can construct a 
family of higher-order mappings from the discrete AKP and BKP equations, by applying the reduction $\tau_{n} \equiv \tau_{Z_{1} k+Z_{2} l+Z_{3} m}$ for any $Z_{1}, Z_{2}$ and $Z_{3}$.

We hope to discuss details of our methods and higher-order mappings in the class given here in a forthcoming paper.

\section{Acknowledgments}

We acknowledge helpful discussions with K Kimura. We gratefully acknowledge financial support from the Australian Research Council Centre of Excellence for Mathematics and Statistics of Complex Systems. KM also acknowledges support from the 21st Century COE program "Development of Dynamic Mathematics with High Functionality" at the Faculty of Mathematics, Kyushu University. KM thanks the department of Mathematics, La Trobe University for warm hospitality.

\section{References}

[1] Quispel G R W , Roberts J A G and Thompson C J 1988 Integrable mappings and soliton equations II Physica D 34 183-192

[2] Ramani A , Grammaticos B and Hietarinta J 1991 Discrete versions of the Painlevé equations Phys. Rev. Lett. 67 1829-1832

[3] Hirota R , Kimura K and Yahagi H 2001 How to find the conserved quantities of nonlinear discrete equations J. Phys. A: Math. Gen. 34 10377-10386

[4] Capel H W and Sahadevan R 2001 A new family of four dimensional symplectic and integrable mappings Physica A289 86-106

[5] Papageorgiou V G, Nijhoff F W and Capel H W 1990 Integrable mappings and nonlinear integrable lattice equations Phys. Lett. 147A 106-114

[6] Quispel G R W, Capel H W, Papageorgiou V G and Nijhoff F W 1991 Integrable mappings derived from soliton equations Physica A173 243-266

[7] Roberts J A G and Quispel G R W, Creating and relating 3-dimensional integrable maps, preprint

[8] Iatrou A 2003 Higher-dimensional integrable mappings Physica D179 229-254

[9] Tuwankotta J M, Quispel G R W and Tamizhmani K M 2004 Dynamics and bifurcations of a threedimensional piecewise-linear integrable map J Phys. A: Math. Gen. 37 12041-12058

[10] Field C M, Nijhoff F W and Capel H W 2005 Exact solutions of quantum mappings from the lattice KdV as multi-dimensional operator difference equations J. Phys. A: Math. Gen. 38 9503-9527

[11] Bellon M P, Maillard J M and Viallet C M 1991 Integrable Coxeter groups Phys. Lett. 159A 221-232

[12] K. Kimura, private communication

[13] Hydon P E 2001 Conservation laws of partial difference equations with two independent variables $J$ Phys. A: Math. Gen. 34 10347-10355

[14] Rasin O G and Hydon P E, Conservation laws of discrete Korteweg-de Vries equations Symmetry, Integrability and Geometry, to appear

[15] Olver P J 1993 Applications of Lie Groups to Differential Equations, Chapter 4, (Springer, New York)

[16] Miwa T 1982 On Hirota's difference equation Proc. Jpn. Acad. A 58 9-12

[17] Hirota R 1981 Discrete Analogue of a Generalized Toda Equation J. Phys. Soc. Jpn. 50 3785-3791 


\section{List of MHF Preprint Series, Kyushu University 21st Century COE Program Development of Dynamic Mathematics with High Functionality}

\section{MHF2003-1 Mitsuhiro T. NAKAO, Kouji HASHIMOTO \& Yoshitaka WATANABE}

A numerical method to verify the invertibility of linear elliptic operators with applications to nonlinear problems

MHF2003-2 Masahisa TABATA \& Daisuke TAGAMI

Error estimates of finite element methods for nonstationary thermal convection problems with temperature-dependent coefficients

MHF2003-3 Tomohiro ANDO, Sadanori KONISHI \& Seiya IMOTO

Adaptive learning machines for nonlinear classification and Bayesian information criteria

MHF2003-4 Kazuhiro YOKOYAMA

On systems of algebraic equations with parametric exponents

MHF2003-5 Masao ISHIKAWA \& Masato WAKAYAMA

Applications of Minor Summation Formulas III, Plücker relations, Lattice paths and Pfaffian identities

MHF2003-6 Atsushi SUZUKI \& Masahisa TABATA

Finite element matrices in congruent subdomains and their effective use for large-scale computations

MHF2003-7 Setsuo TANIGUCHI

Stochastic oscillatory integrals - asymptotic and exact expressions for quadratic phase functions -

MHF2003-8 Shoki MIYAMOTO \& Atsushi YOSHIKAWA

Computable sequences in the Sobolev spaces

MHF2003-9 Toru FUJII \& Takashi YANAGAWA

Wavelet based estimate for non-linear and non-stationary auto-regressive model

MHF2003-10 Atsushi YOSHIKAWA

Maple and wave-front tracking - an experiment

MHF2003-11 Masanobu KANEKO

On the local factor of the zeta function of quadratic orders

MHF2003-12 Hidefumi KAWASAKI

Conjugate-set game for a nonlinear programming problem 
MHF2004-1 Koji YONEMOTO \& Takashi YANAGAWA

Estimating the Lyapunov exponent from chaotic time series with dynamic noise

MHF2004-2 Rui YAMAGUCHI, Eiko TSUCHIYA \& Tomoyuki HIGUCHI

State space modeling approach to decompose daily sales of a restaurant into time-dependent multi-factors

MHF2004-3 Kenji KAJIWARA, Tetsu MASUDA, Masatoshi NOUMI, Yasuhiro OHTA \& Yasuhiko YAMADA

Cubic pencils and Painlevé Hamiltonians

MHF2004-4 Atsushi KAWAGUCHI, Koji YONEMOTO \& Takashi YANAGAWA

Estimating the correlation dimension from a chaotic system with dynamic noise

MHF2004-5 Atsushi KAWAGUCHI, Kentarou KITAMURA, Koji YONEMOTO, Takashi YANAGAWA \& Kiyofumi YUMOTO

Detection of auroral breakups using the correlation dimension

MHF2004-6 Ryo IKOTA, Masayasu MIMURA \& Tatsuyuki NAKAKI

A methodology for numerical simulations to a singular limit

MHF2004-7 Ryo IKOTA \& Eiji YANAGIDA

Stability of stationary interfaces of binary-tree type

MHF2004-8 Yuko ARAKI, Sadanori KONISHI \& Seiya IMOTO

Functional discriminant analysis for gene expression data via radial basis expansion

MHF2004-9 Kenji KAJIWARA, Tetsu MASUDA, Masatoshi NOUMI, Yasuhiro OHTA \& Yasuhiko YAMADA

Hypergeometric solutions to the $q$ - Painlevé equations

MHF2004-10 Raimundas VIDŪNAS

Expressions for values of the gamma function

MHF2004-11 Raimundas VIDŪNAS

Transformations of Gauss hypergeometric functions

MHF2004-12 Koji NAKAGAWA \& Masakazu SUZUKI

Mathematical knowledge browser

MHF2004-13 Ken-ichi MARUNO, Wen-Xiu MA \& Masayuki OIKAWA

Generalized Casorati determinant and Positon-Negaton-Type solutions of the Toda lattice equation

MHF2004-14 Nalini JOSHI, Kenji KAJIWARA \& Marta MAZZOCCO

Generating function associated with the determinant formula for the solutions of the Painlevé II equation 
MHF2004-15 Kouji HASHIMOTO, Ryohei ABE, Mitsuhiro T. NAKAO \& Yoshitaka WATANABE

Numerical verification methods of solutions for nonlinear singularly perturbed problem

MHF2004-16 Ken-ichi MARUNO \& Gino BIONDINI

Resonance and web structure in discrete soliton systems: the two-dimensional Toda lattice and its fully discrete and ultra-discrete versions

MHF2004-17 Ryuei NISHII \& Shinto EGUCHI

Supervised image classification in Markov random field models with Jeffreys divergence

MHF2004-18 Kouji HASHIMOTO, Kenta KOBAYASHI \& Mitsuhiro T. NAKAO

Numerical verification methods of solutions for the free boundary problem

MHF2004-19 Hiroki MASUDA

Ergodicity and exponential $\beta$-mixing bounds for a strong solution of Lévydriven stochastic differential equations

MHF2004-20 Setsuo TANIGUCHI

The Brownian sheet and the reflectionless potentials

MHF2004-21 Ryuei NISHII \& Shinto EGUCHI

Supervised image classification based on AdaBoost with contextual weak classifiers

MHF2004-22 Hideki KOSAKI

On intersections of domains of unbounded positive operators

MHF2004-23 Masahisa TABATA \& Shoichi FUJIMA

Robustness of a characteristic finite element scheme of second order in time increment

MHF2004-24 Ken-ichi MARUNO, Adrian ANKIEWICZ \& Nail AKHMEDIEV

Dissipative solitons of the discrete complex cubic-quintic Ginzburg-Landau equation

MHF2004-25 Raimundas VIDŪNAS

Degenerate Gauss hypergeometric functions

MHF2004-26 Ryo IKOTA

The boundedness of propagation speeds of disturbances for reaction-diffusion systems

MHF2004-27 Ryusuke KON

Convex dominates concave: an exclusion principle in discrete-time Kolmogorov systems 
MHF2004-28 Ryusuke KON

Multiple attractors in host-parasitoid interactions: coexistence and extinction

MHF2004-29 Kentaro IHARA, Masanobu KANEKO \& Don ZAGIER

Derivation and double shuffle relations for multiple zeta values

MHF2004-30 Shuichi INOKUCHI \& Yoshihiro MIZOGUCHI

Generalized partitioned quantum cellular automata and quantization of classical CA

MHF2005-1 Hideki KOSAKI

Matrix trace inequalities related to uncertainty principle

MHF2005-2 Masahisa TABATA

Discrepancy between theory and real computation on the stability of some finite element schemes

MHF2005-3 Yuko ARAKI \& Sadanori KONISHI

Functional regression modeling via regularized basis expansions and model selection

MHF2005-4 Yuko ARAKI \& Sadanori KONISHI

Functional discriminant analysis via regularized basis expansions

MHF2005-5 Kenji KAJIWARA, Tetsu MASUDA, Masatoshi NOUMI, Yasuhiro OHTA \& Yasuhiko YAMADA

Point configurations, Cremona transformations and the elliptic difference Painlevé equations

MHF2005-6 Kenji KAJIWARA, Tetsu MASUDA, Masatoshi NOUMI, Yasuhiro OHTA \& Yasuhiko YAMADA

Construction of hypergeometric solutions to the $q$ - Painlevé equations

MHF2005-7 Hiroki MASUDA

Simple estimators for non-linear Markovian trend from sampled data:

I. ergodic cases

MHF2005-8 Hiroki MASUDA \& Nakahiro YOSHIDA

Edgeworth expansion for a class of Ornstein-Uhlenbeck-based models

MHF2005-9 Masayuki UCHIDA

Approximate martingale estimating functions under small perturbations of dynamical systems

MHF2005-10 Ryo MATSUZAKI \& Masayuki UCHIDA

One-step estimators for diffusion processes with small dispersion parameters from discrete observations

MHF2005-11 Junichi MATSUKUBO, Ryo MATSUZAKI \& Masayuki UCHIDA

Estimation for a discretely observed small diffusion process with a linear drift 
MHF2005-12 Masayuki UCHIDA \& Nakahiro YOSHIDA

AIC for ergodic diffusion processes from discrete observations

MHF2005-13 Hiromichi GOTO \& Kenji KAJIWARA

Generating function related to the Okamoto polynomials for the Painlevé IV equation

MHF2005-14 Masato KIMURA \& Shin-ichi NAGATA

Precise asymptotic behaviour of the first eigenvalue of Sturm-Liouville problems with large drift

MHF2005-15 Daisuke TAGAMI \& Masahisa TABATA

Numerical computations of a melting glass convection in the furnace

MHF2005-16 Raimundas VIDŪNAS

Normalized Leonard pairs and Askey-Wilson relations

MHF2005-17 Raimundas VIDŪNAS

Askey-Wilson relations and Leonard pairs

MHF2005-18 Kenji KAJIWARA \& Atsushi MUKAIHIRA

Soliton solutions for the non-autonomous discrete-time Toda lattice equation

MHF2005-19 Yuu HARIYA

Construction of Gibbs measures for 1-dimensional continuum fields

MHF2005-20 Yuu HARIYA

Integration by parts formulae for the Wiener measure restricted to subsets in $\mathbb{R}^{d}$

MHF2005-21 Yuu HARIYA

A time-change approach to Kotani's extension of Yor's formula

MHF2005-22 Tadahisa FUNAKI, Yuu HARIYA \& Mark YOR

Wiener integrals for centered powers of Bessel processes, I

MHF2005-23 Masahisa TABATA \& Satoshi KAIZU

Finite element schemes for two-fluids flow problems

MHF2005-24 Ken-ichi MARUNO \& Yasuhiro OHTA

Determinant form of dark soliton solutions of the discrete nonlinear Schrödinger equation

MHF2005-25 Alexander V. KITAEV \& Raimundas VIDŪNAS

Quadratic transformations of the sixth Painlevé equation

MHF2005-26 Toru FUJII \& Sadanori KONISHI

Nonlinear regression modeling via regularized wavelets and smoothing parameter selection 
MHF2005-27 Shuichi INOKUCHI, Kazumasa HONDA, Hyen Yeal LEE, Tatsuro SATO, Yoshihiro MIZOGUCHI \& Yasuo KAWAHARA

On reversible cellular automata with finite cell array

MHF2005-28 Toru KOMATSU

Cyclic cubic field with explicit Artin symbols

MHF2005-29 Mitsuhiro T. NAKAO, Kouji HASHIMOTO \& Kaori NAGATOU

A computational approach to constructive a priori and a posteriori error

estimates for finite element approximations of bi-harmonic problems

MHF2005-30 Kaori NAGATOU, Kouji HASHIMOTO \& Mitsuhiro T. NAKAO

Numerical verification of stationary solutions for Navier-Stokes problems

MHF2005-31 Hidefumi KAWASAKI

A duality theorem for a three-phase partition problem

MHF2005-32 Hidefumi KAWASAKI

A duality theorem based on triangles separating three convex sets

MHF2005-33 Takeaki FUCHIKAMI \& Hidefumi KAWASAKI

An explicit formula of the Shapley value for a cooperative game induced from the conjugate point

MHF2005-34 Hideki MURAKAWA

A regularization of a reaction-diffusion system approximation to the two-phase Stefan problem

MHF2006-1 Masahisa TABATA

Numerical simulation of Rayleigh-Taylor problems by an energy-stable finite element scheme

MHF2006-2 Ken-ichi MARUNO \& G R W QUISPEL

Construction of integrals of higher-order mappings 